\title{
RECONSTRUCTION OF THE CARDIA AND FUNDUS OF THE STOMACH
}

\author{
BY \\ GEOFFREY WOOLER \\ From the United Leeds Hospitals
}

(RECEIVED FOR PUbLICATION SEPTEMBER 15, 1956)

This is a preliminary report describing a method of reconstructing the oesophago-gastric junction in order to reproduce the normal valvular mechanism and so prevent peptic oesophagitis. The operation has been performed on three patients, 28, 25, and five months ago. They had peptic ulcers at the cardia, and after these had been resected it was found that in each case the oesophagus was long enough to anastomose again to the stomach in the abdomen. The first patient benefited considerably from the operation and can eat a normal diet; but his lesion was rather complicated and, radiologically, he still has a narrow segment at the site of the oesophagogastric anastomosis. This result is considered partially successful.

The second patient has proved most encouraging. Clinically, radiologically, and at oesophagoscopy he has still a normal functioning "cardia." He has no reflux and no oesophagitis, and is free of symptoms.

The third patient has also done very well. He has no symptoms and is taking a normal diet.

The cardia normally lies inside the abdomen at the lower end of the muscular tunnel forming the oesophageal hiatus of the diaphragm. The circular muscle surrounding the cardia is a continuation of the circular muscle of the oesophagus from which it cannot be differentiated anatomically. It behaves rather differently, however, in so far as it produces a certain degree of sphincteric control. The junction of the oesophageal with gastric mucosa normally lies at the same level as the cardia, but this junction slides up and down during the act of swallowing, for the mucosa is loosely attached to the muscular coats surrounding it.

The cardia may be described as functioning normally when it allows food to pass from the oesophagus into the stomach without delay and prevents gastric contents from regurgitating into the oesophagus.
In my opinion the mechanisms producing this valvular action at the cardia are six.

(1) The cardiac sphincter is the lower segment of the circular muscle of the oesophagus which behaves as a sphincter. Its action is the same if the cardia lies in the chest, associated with a hiatal hernia, or if it prolapses into the abdominal cavity as sometimes happens in cardiospasm.

(2) The obliquity with which the oesophagus enters the stomach is of great importance, and is probably the main factor in preventing reflux. Distension of the stomach decreases this angle, pressing both sides of the oesophagus together, and so obliterates the lumen.

(3) The fundus of the stomach, when it is distended, also presses on the oesophagus and obstructs its lumen. This acts in the same way as a large para-oesophageal hernia, and in this condition reflux of gastric contents into the oesophagus is uncommon.

(4) The right crus of the diaphragm divides to form the hiatal muscle and surrounds the oesophageal hiatus of the diaphragm. Allison (1951) has compared this action to the pubo-rectalis muscle controlling the anal canal. Such a sling, when it contracts, will help to increase the angle between the oesophagus and the stomach.

(5) The oblique muscle fibres of the stomach form another sling of muscle in the stomach wall, which have a similar action to the right crus. Barrett (1954) considers that they are of importance in preventing reflux.

(6) Dornhurst, Harrison, and Pierce (1954) have described a mucosal valve at the cardia, which Nauta (1955) states is a series of mucosal folds surrounding it, and, seen through a gastrostomy opening, he likens it to a rosette.

After considering these various aspects of the mechanism at the cardia, two of them appear to me to be of special importance-namely, the angle 
of entry of the oesophagus into the stomach, and the pressure on the side of the oesophagus by the fundus of the stomach. The operation to be described was designed to restore these two factors, in the hope that they alone would produce a valvular mechanism at the cardia and prevent peptic oesophagitis.

\section{The Choice of Patient}

The ideal type of patient is one who has a gastric ulcer high on the lesser curvature of the stomach which is involving the cardia, or one in which the cardia must be sacrificed in order to excise the ulcer.

Peptic ulceration of the oesophagus is nearly always associated with a hiatal hernia and shortening of the oesophagus. Such patients are better treated after resection of the ulcer by reconstructing the lower oesophagus with an isolated jejunal loop, leaving only the mesentery intact to bring the blood supply. The upper end of this jejunal loop is anastomosed to the divided oesophagus, and the distal end is joined to the posterior wall of the stomach.

If the ulcer is at the bottom of the oesophagus and there is no shortening, re-anastomosis of the oesophagus to the stomach may be attempted after resecting the ulcer, in the manner about to be described.

\section{THE OPERATION}

The patient is placed on the operating table in the left lateral position. The incision begins near to the umbilicus and passes obliquely across the upper abdomen crossing the eighth left costal cartilage and following the seventh intercostal space round behind the vertebral border of the scapula. The entire eighth rib and costal cartilage are resected. The abdominal muscles are incised and the outer border of the rectus sheath is opened, but the rectus muscle is left intact. The peritoneum is opened and also the left pleural cavity. The diaphragm is divided to the oesophageal hiatus. The oesophagus is mobilized from the mediastinum as high as the aortic arch. This may necessitate dividing branches of the vagi, and, as both vagi will be sacrificed when the ulcerated segment of oesophagus is resected, pyloroplasty must be performed. The pyloric sphincter can be divided most easily behind the pylorus. The circular fibres may be felt with the left hand while they are all divided with scissors held in the right. On the posterior aspect of the pylorus this may be achieved without damage to the underlying mucosa.
The ulcerated segment of the lower oesophagus and cardia are resected and removed. Only the divided gastric mucosa is closed with a catgut stitch. A Crafoord clamp is left on the divided oesophagus (Fig. 1).

An opening is made in the anterior wall of the body of the stomach near to the pyloric antrum, through which the inside of the stomach may be inspected.

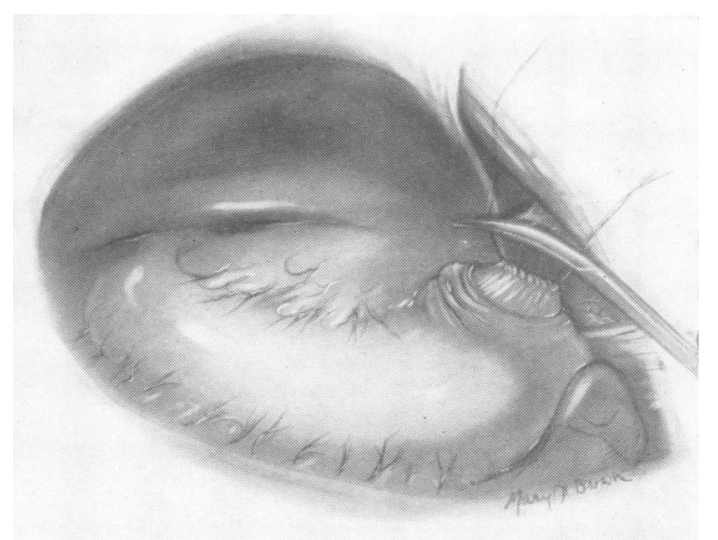

FIG. 1.-The ulcerated and stenosed segment has been excised. The gastric mucosa has been closed and is seen projecting through the gastric muscle which is left open. A clamp secures the divided oesophagus.

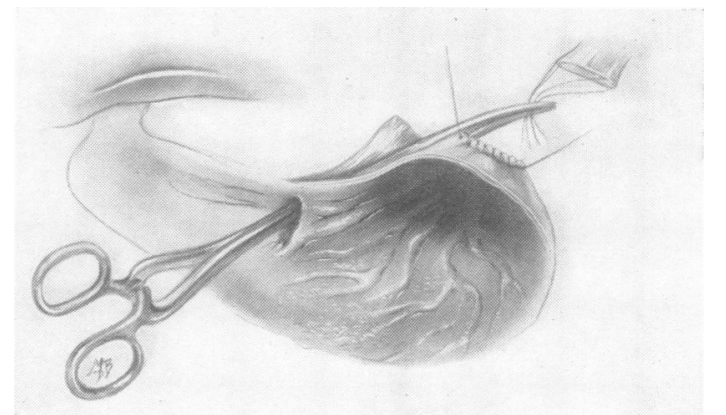

FIG. 2.-A longitudinal section through the stomach, the anterior half of which has been removed. It shows the interior of the stomach and the tunnel along the lesser curvature through which the oesophagus is being drawn.

Returning to where the gastric mucosa has been closed with catgut, a tunnel is made with scissors down the lesser curvature of the stomach between the muscular and mucosal layers of the stomach. At the end of this tunnel an opening is made through the gastric mucosa into the interior of the stomach near to the pyloric end (Fig. 2). The oesophagus is drawn through this tunnel until its end projects into the interior of the stomach (Fig. 3). 
The end of the oesophagus may be secured through the opening in the anterior wall of the stomach, and the oesophageal mucosa anastomosed to the surrounding gastric mucosa (Fig. 4). The opening in the anterior wall of the stomach is then closed. Near to the original cardia the gastric muscle is sutured to the oesophageal muscle, and both are sutured to cover the gastric mucosa previously closed with catgut. This portion of the stomach now forms the new fundus (Fig. 5). The diaphragm is closed, and the wound closed in layers.
CASE 1.-A man, aged 37 years, during May, 1954, perforated a duodenal ulcer which was closed by suture. The oesophageal hiatus of the diaphragm was not examined while the abdomen was open. After the operation the patient developed severe indigestion and heartburn, which recurred intermittently. In June, 1954, he complained of dysphagia ; food appeared to stick in the middle of the chest and sometimes he found that he could not swallow his own saliva. He had lost a few stones in weight.

On clinical examination, apart from the generalized wasting and a healed laparotomy scar, there were no abnormal physical signs. Radiological examination showed a long peptic stricture of the oesophagus

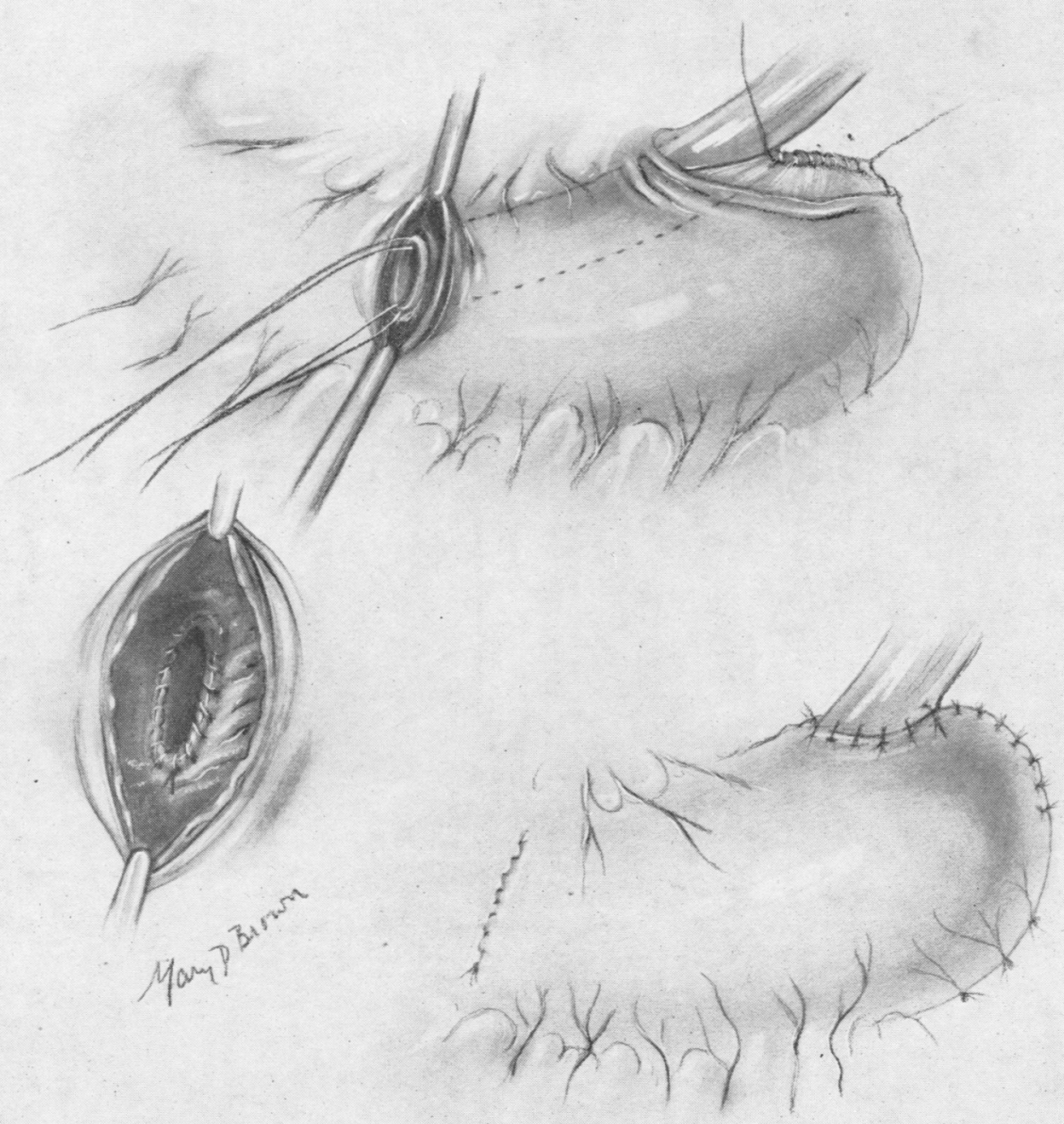

FIG. 3. - Looking through the hole in the anterior wall of the stomach, the oesophagus is seen to be in the correct position. Fig. 4.- Suture of the oesophageal to gastric mucosa.

FIG. 5.-Closure of the anterior gastric opening and of the gastric muscle forming the new fundus of the stomach. 
reaching almost as high as the left main bronchus (Fig. 6), and there was a small sliding hernia allowing free reflux of barium into the oesophagus. A duodenal ulcer was seen and considered to be active.

At oesophagoscopy the instrument could be passed down almost to the cardia without difficulty; the oesophageal mucosa was inflamed and superficially ulcerated, but at $40 \mathrm{~cm}$. (the patient being $6 \mathrm{ft}$. 1 in. tall) a tough fibrous stricture was encountered which dilated with difficulty. There was a small gastric pouch below this stricture, and above it the oesophageal mucosa was ulcerated and inflamed to a distance of $32 \mathrm{~cm}$. from the incisor teeth. The patient was given three months' medical treatment without improvement either radiologically, clinically, or at a further oesophagoscopic examination. Operation was performed on September 2, 1954. A large chronic duodenal ulcer was found producing pyloric obstruction. A Polya type of partial gastrectomy was performed.

In the manner previously described the cardia and the stenosed lower portion of the oesophagus were excised, but on examining the divided end of the oesophagus it was found that fibrosis had spread in the submucous layer for a considerable distance up the oesophagus. If more of the oesophagus had been removed it would not have been possible to join it

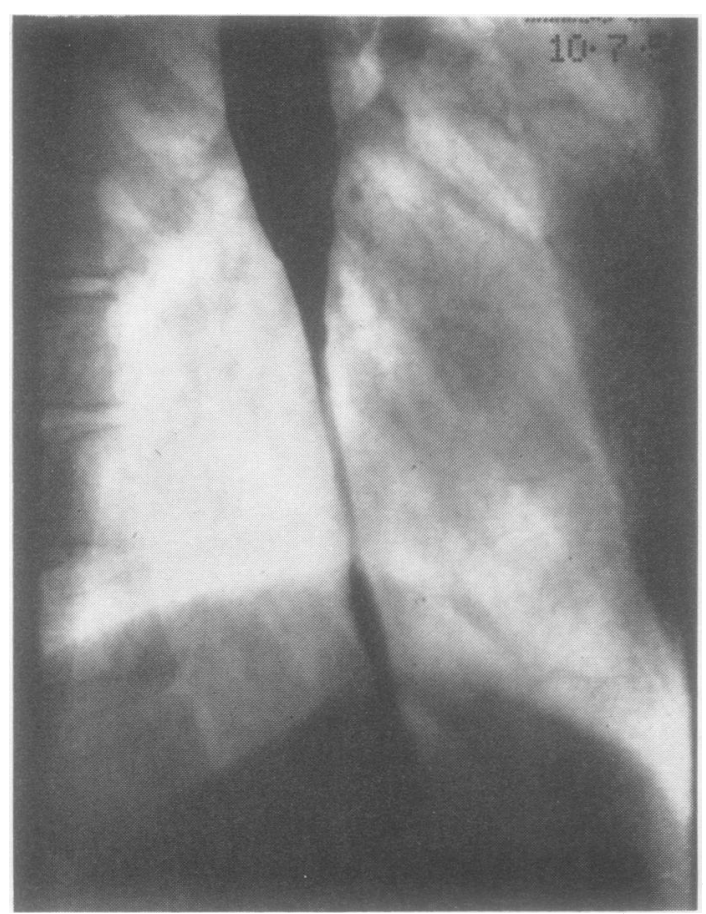

FIG. 6.-Case 1: A pre-operative barium swallow revealed a long narrow segment of oesophagus due to peptic ulceration and reflux oesophagitis associated with a small sliding hiatal hernia. to the stomach. The anastomosis was performed, using this fibrosed portion of oesophagus.

After the operation the patient improved considerably and gained weight. He still has a narrow segment at the site of the anastomosis of the oesophagus to the stomach, which has required dilating three times, but not during the past year. His swallowing is much better than before operation, and he can eat anything providing he eats it slowly. His blood examination is normal and he has had no diarrhoea.

A recent barium swallow shows the narrow segment at the lower end of the oesophagus (Fig. 7), and there is reflux of barium from the stomach into the oesophagus. The patient has been improved clinically, but radiologically he still has an incompetent cardia.

CASE 2.-A man, aged 50, who presented himself in September, 1954, complained of four years' dysphagia. He stated that he could swallow quite well, but having swallowed food it appeared to stick at the lower end of the gullet, and it was some time before he felt it enter the stomach. He had lost over 2 st. in weight.

Radiological examination showed a rather dilated lower segment of the oesophagus which produced vigorous peristalsis. The cardia was closed and barium was forced through an extremely narrow channel into the stomach (Fig. 8).

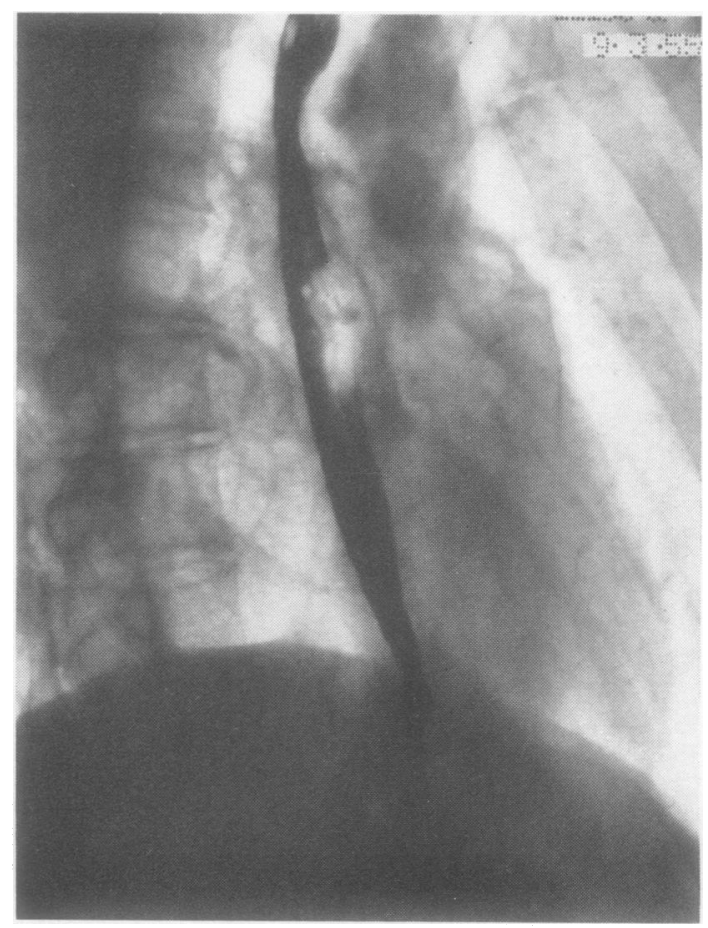

Fig. 7.-Case 1: A barium swallow taken one year after the operation, showing the slightly narrow segment at the site of the new "cardia." 


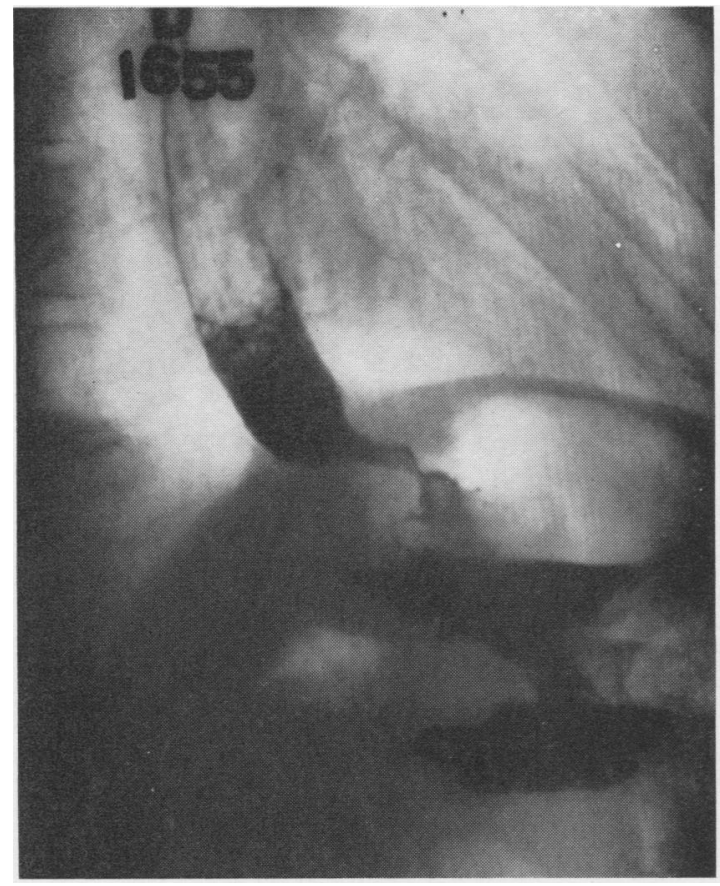

FIG. 8.-Case 2: A chronic gastric ulcer involving the cardia and producing obstruction.

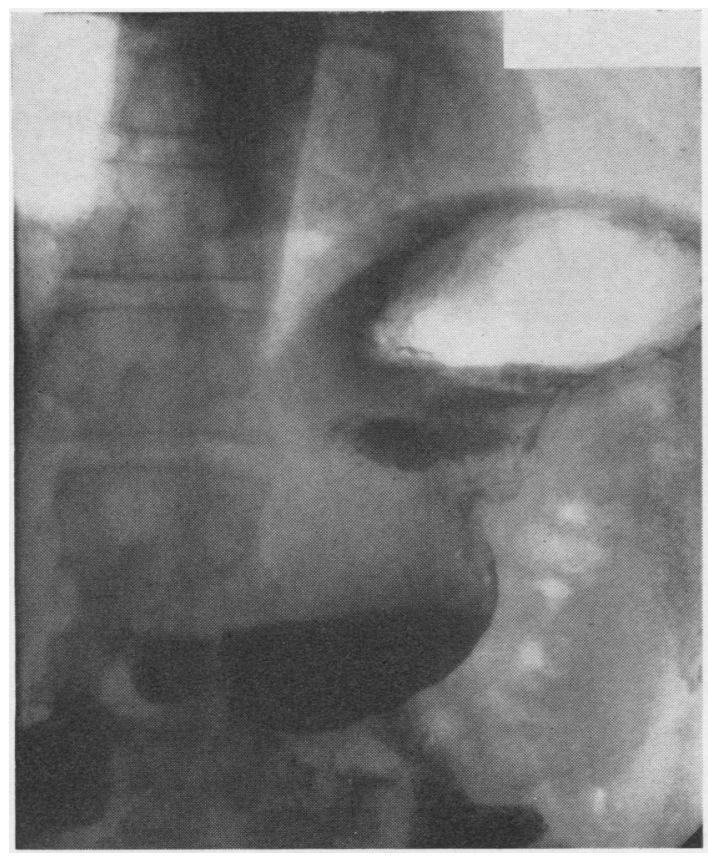

Fig. 10.-Case 3: Pre-operative barium meal showing the large ulcer situated near to the cardia and involving the posterior wall of the stomach.

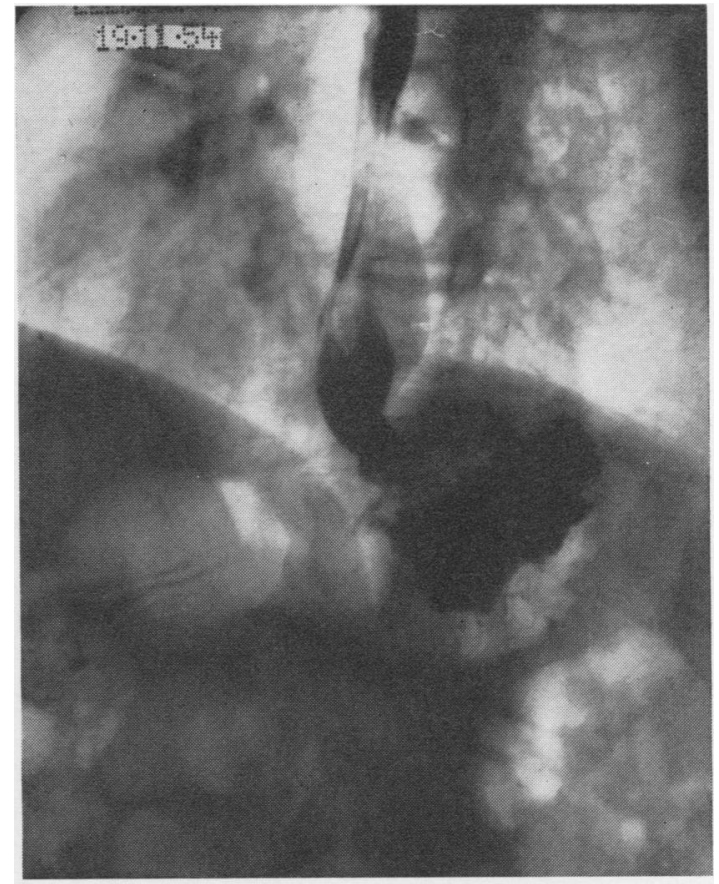

Fig. 9.-Case 2: Reconstruction of the cardia and fundus of the stomach which appears to have produced a normal mechanism. There is no delay to the passage of barium, and reflux of barium back into the oesophagus could not be produced.

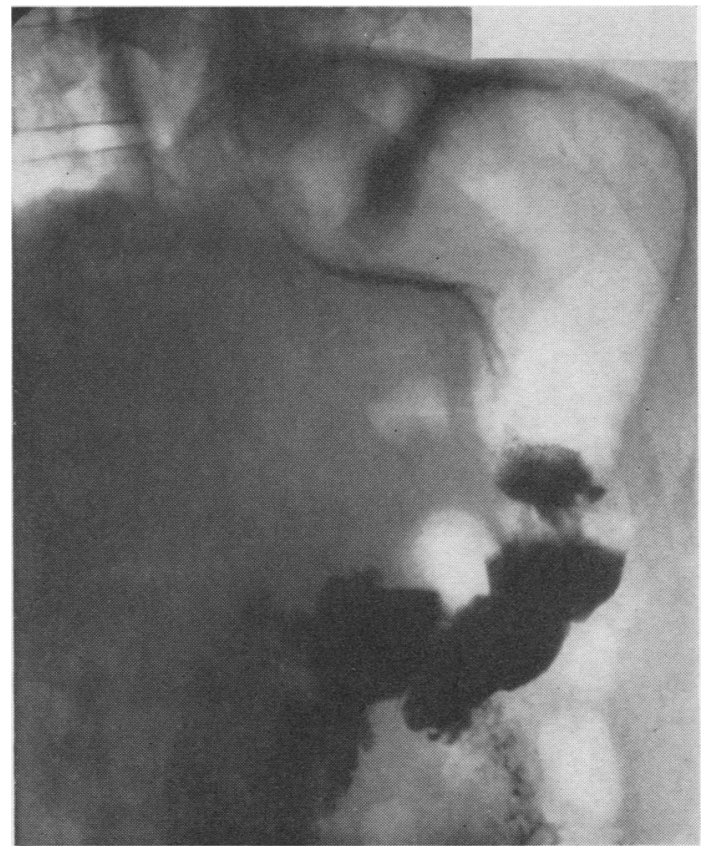

Fig. 11.-Case 3: A barium meal taken four months after reconstruction of the cardia and fundus of the stomach. There was no delay to the passage of barium through into the stomach, but slight reflux could be obtained on abdominal compression. The oesophago-gastric junction looked normal at oesophagoscopy. 
At oesophagoscopy an ulcer was seen surrounding the cardia. The lumen was stenosed, being about $3 \mathrm{~mm}$. in diameter. This stenosis felt hard when an attempt was made to dilate it with bougies.

The operation was performed on September 15, 1954. A chronic gastric ulcer was found surrounding and obstructing the cardia. The oesophagus was not shortened in this case, so after resecting the ulcer it was possible to bring the oesophagus a long way down the lesser curvature of the stomach, between the muscular and mucosal layers, and anastomose it near to the pyloric end of the stomach.

This patient had an uneventful post-operative course. He has gained nearly 3 st. in weight and can eat anything. In December, 1955, he attended a Lord Mayor's banquet, where he ate and drank everything that was provided. He has had no indigestion. Repeated radiological examinations are also satisfactory. Barium flows into the stomach without diffculty and there is no reflux (Fig. 9). A recent oesophagoscopy showed a normal oesophago-gastric junction without oesophagitis. He has in fact a normal cardia, two years after it was reconstructed.

CASE 3.-A man aged 43 gave a three years' history of dyspepsia and epigastric pain occurring about half an hour after meals. He had had three attacks of melaena. Laparotomy was performed on March 23, 1956 , at a regional hospital, and a large mass palpated high on the lesser curvature of the stomach spreading behind the cardia, adherent to the left lobe of the liver and the diaphragm. It was impossible to remove this mass through the abdomen, so the laparotomy wound was closed.

On April 6 he was transferred to the General Infirmary at Leeds. A barium meal was performed which showed a large ulcer on the posterior wall just below the cardia and extending from the lesser curvature to the fundus of the stomach (Fig. 10). There was also an irregularity of the mucosa noted at the junction of the oesophagus and cardia. The lesion was thought to be malignant.

Operation was performed on April 17, 1956, through a left thoraco-abdominal incision, which gave access to the lower oesophagus and the upper abdomen. The ulcer was very large but looked inflammatory, and a frozen histological section confirmed this belief. It had perforated into the left lobe of the liver and the right crus of the diaphragm. It involved the cardia, and the edge of the ulcer reached one side of the oesophageal wall. There was no apparent way of resecting it without removing the cardia. The oesophagus was therefore divided above the cardia, and the ulcerated portion of stomach removed.

The divided oesophagus was re-anastomosed to the stomach and pyloromyotomy performed. He has had no dysphagia or digestive symptoms since this operation, but his post-operative course was complicated by two pulmonary emboli from phlebitis of a calf vein which responded to anticoagulant therapy. A recent barium swallow (Fig. 11) showed that the oesophago-gastrostomy was working satisfactorily. Barium passed rapidly into the stomach without delay, and there was no hold-up at the site of the new "cardia." With the patient in the Trendelenburg's position and on abdominal compression some barium regurgitated into the oesophagus. Oesophagoscopic examination was performed which revealed no oesophagitis ; the instrument passed round an angle as it entered the stomach through the new cardia, which looked anatomically correct. The patient is gaining weight and enjoying a normal diet.

\section{SUMMARY}

A method of re-anastomosing the oesophagus to the stomach has been described, which has been successful in reproducing the normal mechanism of the cardia. After excision of an ulcer near the cardia, and if there is little or no shortening of the oesophagus, this method may be used to join the oesophagus to the stomach.

My thanks are due to Professor A. S. Johnstone, who performed the radiological examinations of these patients.

\section{REFERENCES}

Allison, P. R. (1951). Surg. Gynec. Obstet., 92, 419.

Barrett, N. R. (1954). Brit. J. Surg., 42, 231.

Dornhorst, A. C., Harrison, K., and Pierce, J. W. (1954). Lancet, 1,695 .

Nauta, J. (1955). Een studie van het afsluitingsmechanisme tussen slokdarm en maag. 